\title{
Using nonlinear jumps to estimate cubic stiffness nonlinearity: An experimental study
}

Proc IMechE Part C:

$J$ Mechanical Engineering Science

0(0) 1-7

(C) IMechE 2015

Reprints and permissions:

sagepub.co.uk/journalsPermissions.nav DOI: 10.1 I77/09544062I5606746

pic.sagepub.com

\author{
Bin Tang', MJ Brennan', V Lopes Jr², S da Silva² and R Ramlan ${ }^{3}$
}

\begin{abstract}
Attempts are being made to improve mechanical design by using nonlinearity rather than eliminating it, especially in the area of vibration control and in energy harvesting. In such systems, there is a need to both predict the dynamic behavior and to estimate the system properties from measurements. This paper concerns an experimental investigation of a simple identification method, which is specific to systems in which the behavior is known to be similar to that of a Duffing-type system. It involves the measurement of jump-down frequencies and the amplitudes of displacement at these frequencies. The theoretical basis for the method is briefly described as, is an experimental investigation on a beamshaker system. The results are comparable with those determined by the restoring force surface method. The method described in this article has the advantage that the data can be collected and processed more easily than the restoring force surface method and can be potentially more suitable for the engineering community than existing identification measures.
\end{abstract}

\section{Keywords \\ Cubic stiffness nonlinearity, parameter estimation, jump phenomena, stepped-sine, least-square fitting}

Date received: 7 May 2015; accepted: 26 August 2015

\section{Introduction}

Many engineering structures behave as nonlinear systems for high levels of excitation because of geometric stiffness. ${ }^{1}$ Clamped-clamped beams, cable-wire isolators, and micromachined resonators ${ }^{1-4}$ are examples of such structures, which have a restoring force that is dependent upon the cube of the displacement for relatively large amplitude motion-so-called Duffing-type systems. Such systems may have jumpup and jump-down frequencies depending upon the level of damping. ${ }^{5}$ There is a need to validate theoretical models of nonlinear systems, which involves experimental determination of the system properties.

There is extensive literature on nonlinear system identification, and a comprehensive review on this topic has been provided by Kerschen et al. ${ }^{2}$ System identification generally involves excitation of the structure and measuring the response; if possible the excitation force is also measured. The Hilbert transform can be used on the free vibration signal to determine some properties of the system, for example from the backbone curve. ${ }^{6}$ There are several methods to determine the system properties using data from tests involving random excitation, such as the reverse path method, ${ }^{7}$ consideration of the nonlinear forces as feedback forces, ${ }^{8}$ nonlinear subspace identification, ${ }^{9}$ and use of the Volterra series. ${ }^{10}$ Recently, a globallocal nonlinear system identification method, ${ }^{11}$ a frequency response function method, ${ }^{12,13}$ time- and frequency-domain subspace methods, ${ }^{14}$ synchronization-based method, ${ }^{15}$ and resonance decay method ${ }^{16}$ have been used to estimate strong nonlinearity in single or multiple degree-of-freedom (MDOF) systems. There are many other methods, and the reader is referred to Worden and Tomlinson ${ }^{17}$ and the references therein for further details.

This article is concerned with experimental verification of a simple frequency domain method of determining system parameters, ${ }^{18,19}$ which may be of interest to practicing engineers. It falls into the same category as the frequency domain methods discussed by Carrella and Ewins ${ }^{12}$ and Arslan et al. ${ }^{13}$ These

\footnotetext{
'Institute of Internal Combustion Engine, Dalian University of Technology, Dalian, China

${ }^{2}$ Departamento de Engenharia Mecânica, Univ Estadual Paulista (UNESP), Ilha Solteira, São Paulo, Brasil

${ }^{3}$ Faculty of Mechanical Engineering, Universiti Teknikal Malaysia Melaka, Hang Tuah Jaya, Melaka, Malaysia
}

\section{Corresponding author:}

Bin Tang, Institute of Internal Combustion Engine, Dalian University of Technology, Dalian I 16023, China.

Email: btang@dlut.edu.cn 
methods are distinctive in that they are relatively simple to implement and can be related in some sense to system identification of linear vibrating systems. Of course, because of their simplicity these methods have some limitations. For example, the method discussed by Carrella and Ewins ${ }^{12}$ is suitable for single-degree-of-freedom (SDOF) systems with weak nonlinearities such that jumps do not occur in the system response. The basic principle of the first method discussed by Arslan et al. ${ }^{13}$ is similar to that discussed by Carrella and Ewins ${ }^{12}$, but can, in principle, be applied to MDOF systems. The disadvantage is that a controller is required in the experimental setup to control the output of the shakers so that the structural response is the same at all frequencies. The second method in Arslan et al. ${ }^{13}$ uses the first method and then proposes a simple way of determining the describing function (which is the stiffness of the system in the case of a stiffness only nonlinearity). In the methods described by Carrella and Ewins ${ }^{12}$ and Arslan et al., ${ }^{13}$ multiple nonlinearities of both stiffness and damping can be accommodated. In the method described in this paper, a SDOF system with cubic stiffness nonlinearity is addressed. Accordingly, it is a grey-box approach, which is applicable when the type of system nonlinearity is known a priori. The advantage of the approach is that it is very simple, based on clear physical principles so that it is easy to visualize the way in which it works, and can be conducted without the need for sophisticated test equipment or computing power. This is contrary to many of the methods described recently in the literature, which tend to be methods that do not require knowledge of the physical behavior of the system (black box), and can be used for multiple types of nonlinearities. Accordingly, they tend to be complex, and the ways in which they work in terms of the physics rather than the mathematics is not very transparent.

The method described in this paper is based on the estimation of the mass of the system from low-level random excitation, and then the determination of the linear and nonlinear stiffness parameters by exciting the system at discrete frequencies so that the jumpdown frequency can be identified for a several levels of excitation. The linear and nonlinear stiffness parameters are then estimated from this data and compared with those determined using the restoring force surface (RFS) method. ${ }^{17}$

\section{Parameter estimation}

If the vibration modes of a structure are well-separated, then the first mode of vibration can be considered as a SDOF system. An example of such a system, which is of interest here, is a uniform straight beam, which is constrained at the ends and is subject to various levels of excitation. For low levels of vibration the dominant stiffness is from bending, and the system behaves as a linear structure, but for higher levels of vibration the in-plane stretching of the beam contributes to the stiffness, which results in a nonlinear stiffness. ${ }^{1}$ The first mode of this system can be described as a Duffing-type SDOF system whose equation of motion, for harmonic excitation, is given by

$$
m \ddot{x}+c \dot{x}+k_{1} x+k_{3} x^{3}=F \cos (\omega t)
$$

where $m$ is the mass, $c$ is the damping coefficient, $k_{1}$ and $k_{3}$ are the linear and nonlinear stiffness coefficients respectively, and $F$ is the amplitude of the harmonic excitation force; the overdots represent differentiation with respect to time. Introducing the non-dimensional parameters

$$
\begin{gathered}
x_{0}=\frac{m g}{k_{1}}, \quad y=\frac{x}{x_{0}}, \quad \gamma=\frac{k_{3} x_{0}^{2}}{k_{1}}, \quad \zeta=\frac{c}{2 m \omega_{n}}, \\
\omega_{n}^{2}=\frac{k_{1}}{m}, \quad \tau=\omega_{n} t, \quad \Omega=\frac{\omega}{\omega_{n}}, \quad \hat{F}=\frac{F}{m g}
\end{gathered}
$$

Equation (1a) can be written as

$$
y^{\prime \prime}+2 \zeta y^{\prime}+y+\gamma y^{3}=\hat{F} \cos (\Omega \tau)
$$

where $(\bullet)^{\prime}$ denotes differentiation with respect to $\tau$, and the static displacement $x_{0}$ of the system due to its own weight is used to nondimensionalize the displacement. Applying the harmonic balance method (HBM) and assuming a solution of the form $y=Y \cos (\Omega \tau+\phi)$ results in the frequency-amplitude relationship given by ${ }^{5}$

$$
\Omega_{1,2}=\left(\begin{array}{c}
1-2 \zeta^{2}+\frac{3}{4} \gamma Y^{2} \\
\pm\left(-4 \zeta^{2}\left(1-\zeta^{2}+\frac{3}{4} \gamma Y^{2}\right)+\frac{\hat{F}^{2}}{Y^{2}}\right)^{\frac{1}{2}}
\end{array}\right)^{\frac{1}{2}}
$$

For a lightly-damped system in which $\zeta^{2} \ll 1$, the relationship between the jump-up $\Omega_{u}$, and jump-down $\Omega_{d}$ frequency and the nonlinear stiffness are respectively given $b^{5}$

$$
\begin{aligned}
& \Omega_{u}^{2} \approx 1+\left(\frac{3}{2}\right)^{\frac{4}{3}}\left(\gamma \hat{F}^{2}\right)^{\frac{1}{3}} \\
& \Omega_{d}^{2} \approx \frac{1}{2}\left(1+\left(1+\frac{3 \gamma \hat{F}^{2}}{4 \zeta^{2}}\right)^{\frac{1}{2}}\right)
\end{aligned}
$$

From equation (2), the jump-down frequency is also related to the nonlinear stiffness by

$$
\Omega_{d}^{2} \approx 1-2 \zeta^{2}+\frac{3}{4} \gamma Y_{d}^{2}
$$


From equations (3a) to (3c) the nonlinear parameter can be estimated by

$$
\gamma \approx \frac{1}{\hat{F}^{2}}\left(\frac{2}{3}\right)^{4}\left(\Omega_{u}^{2}-1\right)^{3}
$$

or

$$
\gamma \approx \frac{4 \zeta^{2}}{3 \hat{F}^{2}}\left(\left(2 \Omega_{d}^{2}-1\right)^{2}-1\right)
$$

or

$$
\gamma \approx \frac{4}{3 Y_{d}^{2}}\left(\Omega_{d}^{2}-1+2 \zeta^{2}\right)
$$

Note that if equation (4a) is used then the jump-up frequency and the nondimensional excitation force need to be known. If equation (4b) is used then the jump-down frequency, the nondimensional excitation force and the damping ratio need to be known. If equation (4c) is used then the jump-down frequency, the amplitude of the response at the jumpdown frequency and the damping ratio need to be known. If the damping is light and the nonlinearity strong enough so that $\Omega_{d}^{2}-1 \gg 2 \zeta^{2}$ then damping can be neglected in equation (4c).

An issue with using equations (4a) to (4c) is the sensitivity of the nonlinear stiffness estimate to errors that occur in the measurement of the jump frequencies. The percentage error in the estimation of the nondimensional stiffness parameter is plotted as a function of the nondimensional jump-up frequency in Figure 1(a) and the corresponding percentage errors as a function of the jump-down frequencies, estimated using equations (4b) and (4c), are shown in Figure 1(b) and (c), respectively. Errors in the estimate of the jump frequency from $1 \%$ to $5 \%$ are considered and damping in equation (4c) is neglected. It is clear from Figure 1(a) to (c) that in all cases the error in the estimate of the nonlinear stiffness using jump frequencies is potentially large.

To minimize the potential error from a single measurement, several measurements are made for different levels of excitation, and equation (3c) is used to give an estimate of the nonlinearity. In equation (3c), it is clear if $\Omega_{d}^{2}$ is plotted for a corresponding value of $Y_{d}^{2}$ for each excitation level, then provided that the system under test behaves as a Duffing oscillator with linear viscous damping, the resulting data points should lie on a straight line, with the slope of this line being $3 \gamma / 4$. Note that equation (3c) is valid even if the excitation is not high enough for a jumpdown frequency to occur. In this case the points of interest are the resonance frequency and the displacement amplitude at this frequency. The intersection of the line with the $\Omega_{d}^{2}$ axis should coincide with the nondimensional resonance frequency of the underlying linear system. In practice, the points will not lie
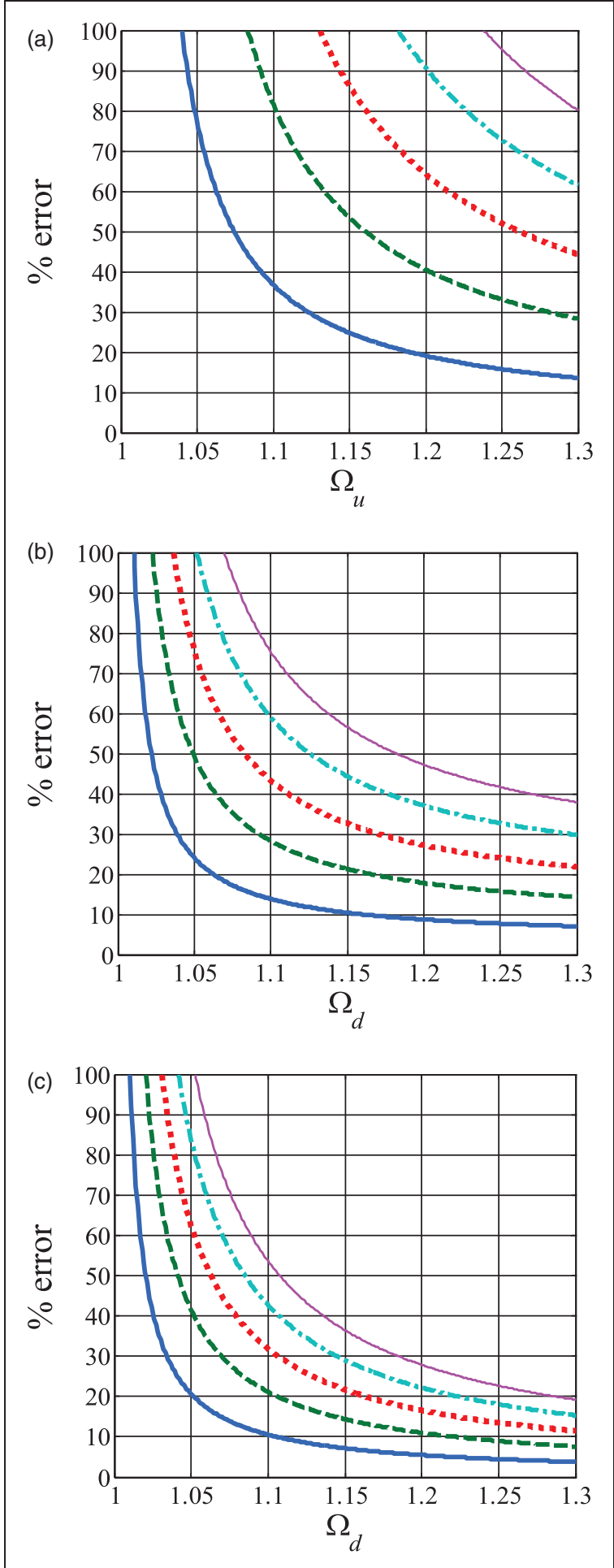

Figure I. Percentage error in the estimate of the nondimensional nonlinear stiffness parameter as a function of the jump frequency. (a) Using equation (4a), (b, c) using equations (4b) and (4c) respectively. Errors in the measurement of the jump frequency, thick solid line $1 \%$, dashed line $2 \%$, dotted line $3 \%$, dashed-dotted line $4 \%$, and thin solid line $5 \%$.

perfectly on straight line, so a straight-line, leastsquares fit to the data points is necessary. The intersect of this line with the $y$ axis gives the damped natural frequency of the underlying linear system, which can be used to determine the linear stiffness. 
Either before or after $\gamma$ has been estimated, the mass and damping can be determined. This can be done by measuring the receptance using low-level random excitation, so that the nonlinear stiffness will have a negligible effect compared to the linear stiffness. If the damping is light $(\zeta<0.1)$, the damping ratio can be estimated by measuring the half-power bandwidth of the mode, $\Delta \omega,{ }^{20}$ and then the damping ratio can be determined by $\zeta \approx \Delta \omega /\left(2 \omega_{n}\right)$. Now, the dynamic stiffness of the system is the reciprocal of the receptance and is given by $k_{1}-\omega^{2} m+j \omega c$. If the real part of this is plotted as a function of the square of the excitation frequency, then the slope of this line is $-m$. Once the mass and damping ratio has been determined, the linear stiffness $k_{1}$ can be estimated using the damped natural frequency. Finally, the nonlinear stiffness can be determined from $k_{3}=\gamma k_{1} / x_{0}^{2}=\gamma \omega_{n}^{6} \mathrm{~m} / \mathrm{g}^{2}$.

\section{Experimental work}

The test-rig used in this work is shown in Figure 2. It consists of a compressed clamped-clamped aluminum beam to give a structure with a hardening nonlinear cubic stiffness, a TIRA shaker, and a DYTRAN accelerometer. The beam has a width of $20 \mathrm{~mm}$, a thickness of $1.8 \mathrm{~mm}$, and a length of $460 \mathrm{~mm}$. The mass of the beam is $50 \mathrm{~g}$. The exact details of the structure are relatively unimportant, the main point being that the combined beam-shaker system acted as an SDOF oscillator over a limited frequency range. For all the tests, the voltage signal to the amplifier driving the shaker was used as the reference source. This was done as it was relatively easy to keep the current supplied to the shaker constant over a range of frequencies (using the amplifier in current mode), which was not the case for the force applied to the beam.

A compressive axial force was applied to the beam using a screw at the top of the test-rig. It was applied gradually until the beam buckled and then the screw was rotated in an anti-clockwise direction until the beam was just on the point of buckling. As shown in Kovacic and Brennan, ${ }^{1}$ the compression force has the effect of reducing the linear stiffness of the beam so that the nonlinear hardening stiffness has a more pronounced effect compared to the linear stiffness.

Before conducting experiments on the test-rig, the relationship between the source voltage supplied to the amplifier and the blocked force generated by the shaker was determined by dynamic calibration of the shaker. It was found to be $9.9 \mathrm{~N} / \mathrm{V}$. Following the calibration of the shaker, the beam-shaker system was excited by low-level random noise so that it behaved as a linear system. The estimated mass and damping ratio of the underlying linear system were estimated using the method described in "Parameter estimation" section and are given in Table 1.

Tests to determine the nonlinear stiffness were then conducted using stepped-sine excitation. The excitation frequency was increased from $20 \mathrm{~Hz}$ to $50 \mathrm{~Hz}$ in $1 \mathrm{~Hz}$ steps, and seven different levels of excitation were used. In each case, the source signal to the amplifier was kept constant and hence the force applied to the beamshaker system, was kept constant. The data was collected using an $\mathrm{m}+\mathrm{p}$ VibPilot data acquisition system.

The frequency response curves (FRCs) for the centre of the beam for different levels of excitation (a)

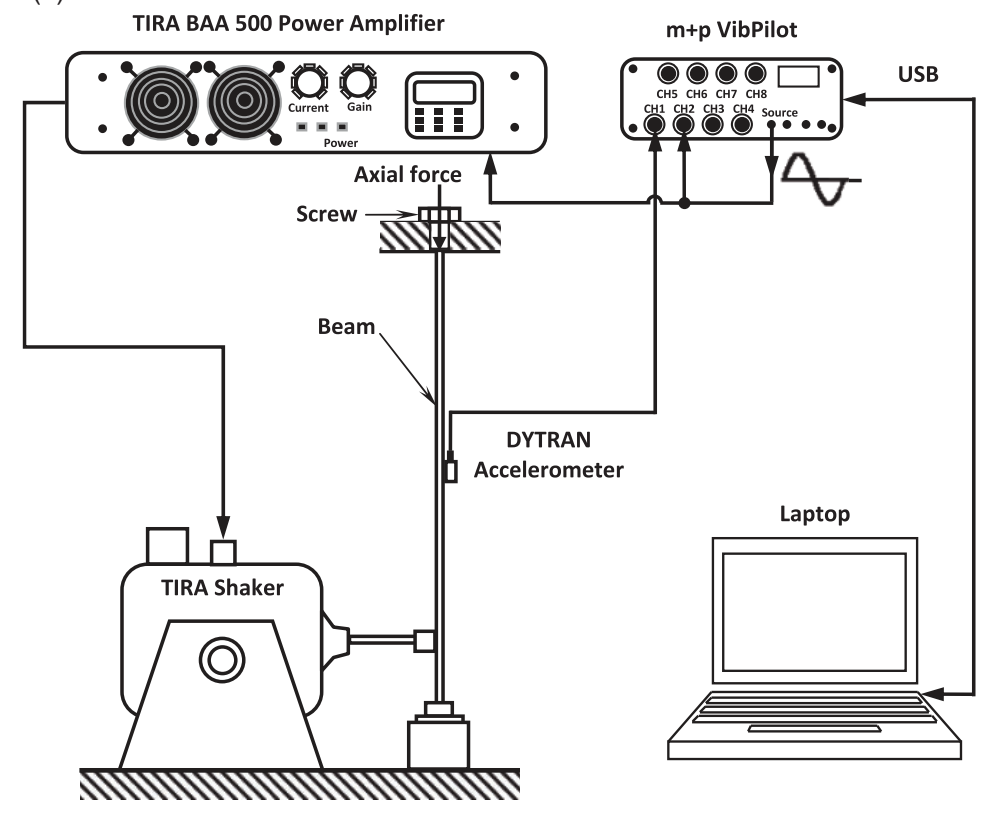

(b)

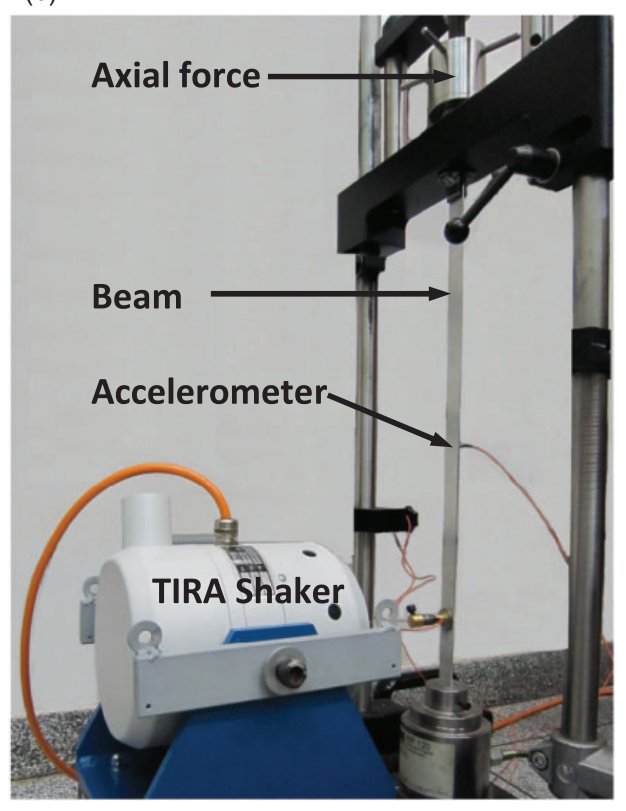

Figure 2. Experimental setup for the measurement of the beam-shaker system. 
Table I. Estimated parameters.

\begin{tabular}{lllll}
\hline Mass $(\mathrm{kg})$ & $\begin{array}{l}\text { Damping } \\
\text { ratio }\end{array}$ & & $k_{1}(\mathrm{~N} / \mathrm{m})$ & $k_{3}\left(\mathrm{~N} / \mathrm{m}^{3}\right)$ \\
\hline 0.141 & 0.0187 & Current method & 6830 & $4.78 \times 10^{8}$ \\
& & RFS method & 7237 & $3.93 \times 10^{8}$ \\
\hline
\end{tabular}

RFS: restoring force surface.

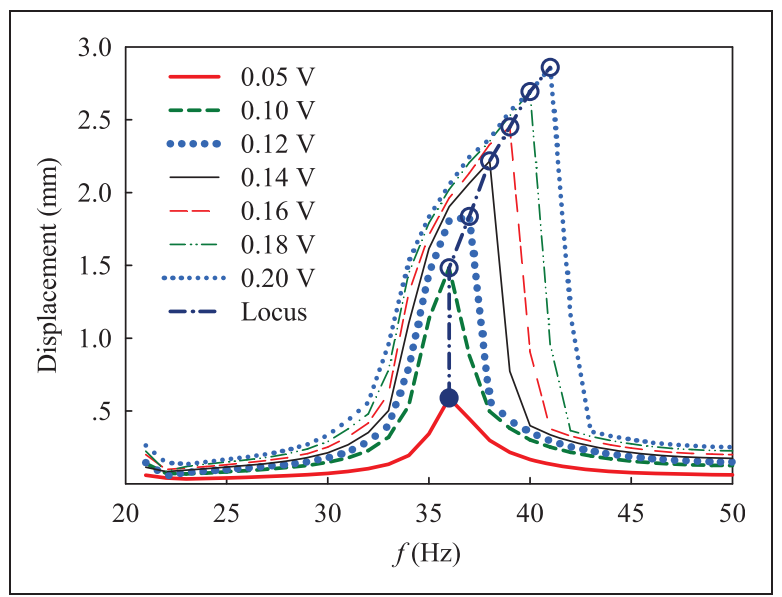

Figure 3. Measured displacement of the middle point of the beam and the locus of the points from either the peak or the jump point; $\bigcirc$, jump-down point; $\bullet$, peak point.

are shown in Figure 3. The jump-down points are indicated on the figure, together with the point of maximum amplitude for low level of excitation when no jump occurred.

\section{Estimation of the nonlinear stiffness}

To determine the nonlinear cubic stiffness, equation (3c) can be written in dimensional form as

$$
f_{d}^{2} \approx f_{r}^{2}+\frac{3}{16 \pi^{2}} \frac{k_{3}}{m} X_{d}^{2}
$$

where $f_{r}=f_{n} \sqrt{1-2 \zeta^{2}}$ is the frequency at which the peak occurs in the displacement response of a damped linear oscillator, $f_{n}=\omega_{n} /(2 \pi)$ and $X_{d}$ is the displacement amplitude of the response at either the approximated jump-down frequency (or the maximum response when a jump does not occur). To determine $k_{3}$ from the slope of the graph of $f_{d}^{2}$ as a function of $X_{d}^{2}$ only the mass needs to be known, which was determined previously.

The results are plotted in Figure 4. Two results are shown as the jump-down frequency is not known exactly. This is because although the jump occurs at a specific frequency in the test, it may, in principle, occur at some point between that frequency and the next frequency step. The circles denote the measured amplitudes at the jump-down frequencies, while the

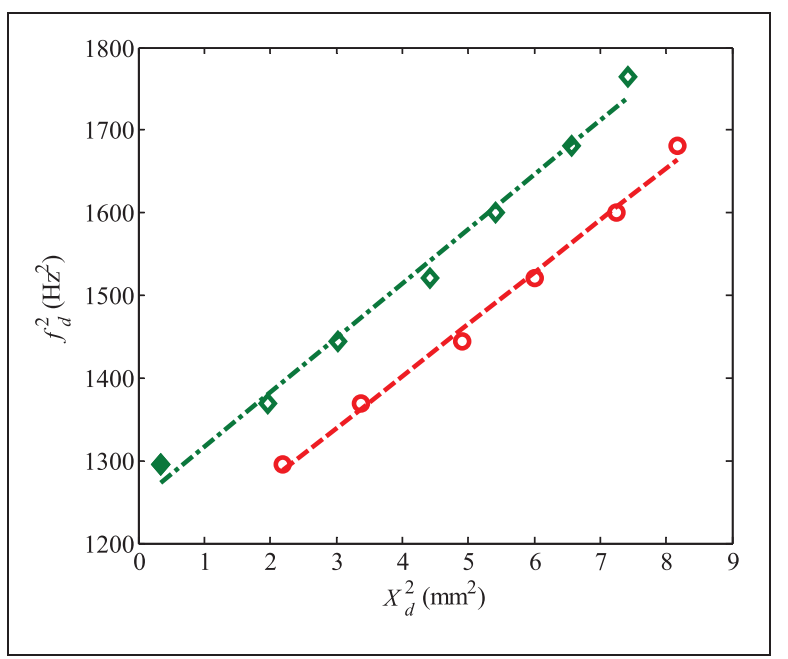

Figure 4. Plot used to determine the nonlinear stiffness $k_{3}$ from experimental data. $\bigcirc$ is the jump-down point, $\diamond$ and $\diamond$ denote the jump-down and peak points with the jump frequency incremented by $\mathrm{I} \mathrm{Hz}$. The straight lines are fitted to the data in a least-squares sense.

diamonds denote the measured amplitudes with the jump-down frequencies increased by $1 \mathrm{~Hz}$ from the measured jump frequencies. As shown in Figure 3, the solid marker in Figure 4 denotes the peak in the response and the hollow markers denote the jumpdown points. As acceleration was measured, the amplitude of the displacement at the jump-down frequency was determined by dividing the value of the acceleration at the jump-down frequency by $\left(2 \pi f_{d}\right)^{2}$. Once the gradients of the lines were calculated, the values of the nonlinear stiffness could be determined. They were found to be $4.65 \times 10^{8} \mathrm{Nm}^{-3}$ (dashed line) and $4.90 \times 10^{8} \mathrm{Nm}^{-3}$ (dashed-dotted line), which results in an average nonlinear stiffness of $4.78 \times 10^{8} \mathrm{Nm}^{-3}$.

To check the nonlinear stiffness estimate it is compared with the estimate from the RFS method. ${ }^{17}$ The system was excited with a swept-sine signal, and the excitation force and acceleration of the center of the beam were measured. To apply the RFS method equation (1a) is rearranged to give

$$
c \dot{x}+k_{1} x+k_{3} x^{3}=f(t)-m \ddot{x}
$$

The right-hand side of equation (6) is known, as the acceleration and mass are measured, as is the excitation force $f(t)$. The velocity and the displacement are determined by time-domain integration of the acceleration signal. A three-dimensional surface $(x, \dot{x}, f(t)-m \ddot{x})$ can then be plotted using the data at each measured time instant. A section through the surface is then extracted between the values of $-0.005 \mathrm{~m} / \mathrm{s}<\dot{x}<0.005 \mathrm{~m} / \mathrm{s}$. This is plotted in Figure 5 as the restoring force. A polynomial is subsequently fitted to the data to give the linear and nonlinear stiffness which are $k_{1}=7237 \mathrm{~N} / \mathrm{m}$ and $k_{3}=3.93 \times 10^{8} \mathrm{~N} / \mathrm{m}^{3}$. 


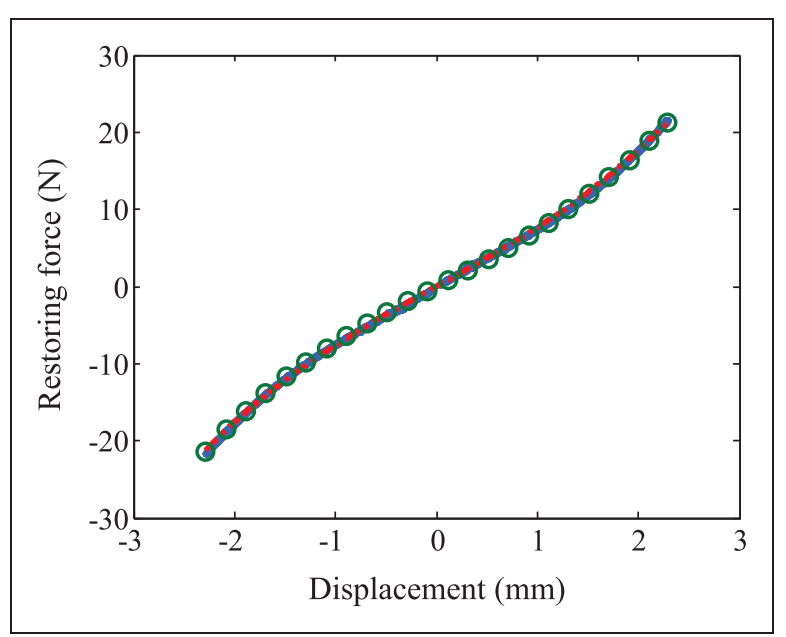

Figure 5. Estimated restoring force curves using the current method (green circle) and RFS method (dashed-dotted red line); solid blue line, measured restoring spring force curve.

Table 2. Comparison of the current method with the RFS method.

\begin{tabular}{lll}
\hline & Current method & RFS Method \\
\hline Methodology & Grey-box & Black-box \\
Domain & Frequency & Time \\
Nonlinearity & Low and Strong & Low and Strong \\
Measurement & Simple & Simple \\
Measurement times & Multiple & Single \\
Measurement time & Short & Short \\
Signal processing & Simple & Complex \\
Prior parameters & Mass, Damping & Mass, Damping \\
Prior parameters effect & High & High \\
Numerical method & Linear fitting & Polynomial fitting \\
\hline
\end{tabular}

RFS: restoring force surface.

The estimated parameters are given in Table 1. Comparing the stiffness results obtained using the current and RFS methods, it can be seen that the difference between linear stiffness is about $6 \%$, while the difference between the nonlinear stiffness is about $20 \%$. Although there are some differences between the estimated parameters, the two estimated restoring force curves are very similar when they are plotted together, as can be seen in Figure 5. A summary of the two methodologies is compared in Table 2. It can be seen that the two methods are both simple and physical. If possible, both methods are suggested to be used to estimate the nonlinearity and compared with each other.

\section{Conclusions}

This article has described an experimental investigation into a method to estimate the cubic stiffness nonlinearity in a Duffing-like system. The method is based on exciting the system over a range of amplitudes and frequencies and measuring the jump-down frequencies and corresponding amplitude at these frequencies. It has been shown that whilst in principle the nonlinear stiffness can be estimated from a single measurement, the error from this measurement can potentially be large. It is, therefore, preferable to excite the system over a range of amplitudes. The stepped-sine excitation method was used to estimate the jump-down points at different excitation amplitudes. The estimated linear and nonlinear stiffness of the system are compared with those determined from the RFS method (which compare reasonably well). The advantage of the approach is that it is simple, based on clear physical principles so that it is easy to visualize the way in which it works, and can be conducted easily without the need for sophisticated test equipment or computing power.

\section{Declaration of Conflicting Interests}

The author(s) declared no potential conflicts of interest with respect to the research, authorship, and/or publication of this article.

\section{Funding}

This work was supported by the National Natural Science Foundation of China (NSFC) (grant number 11202048), the Fundamental Research Funds for the Central Universities of China (grant number DUT15QY30), Brazilian National Council for Scientific and Technological Development (CNPq) (grant number 401360/2012-1), and São Paulo Research Foundation (FAPESP) (grant number 12/09135-3).

\section{References}

1. Kovacic I and Brennan MJ. The Duffing equation: Nonlinear oscillators and their behaviour. Chichester: Wiley, 2011. Chaps. 2, 8.

2. Kerschen G, Worden K, Vakakis AF, et al. Past, present and future of nonlinear system identification in structural dynamics. Mech Syst Signal Process 2006; 20: 505-592.

3. Budnitzki M, Scates MC, Ritchie RO, et al. The effects of cubic stiffness on fatigue characterization resonator performance. Sens Actuat A: Phys 2010; 157: 228-234.

4. Wiebe R and Spottswood SM. Co-existing responses and stochastic resonance in post-buckled structures: A combined numerical and experimental study. J Sound Vib 2014; 333: 4682-4694.

5. Brennan MJ, Kovacic I, Carrella A, et al. On the jumpup and jump-down frequencies of the Duffing oscillator. J Sound Vib 2008; 318: 1250-1261.

6. Feldman M. Hilbert transform in vibration analysis. Mech Sys Signal Process 2011; 25: 735-802.

7. Bendat JS. Nonlinear system analysis and identification from random data. New York: Wiley, 1990.

8. Adams DE and Allemang RJ. A frequency domain method for estimating the parameters of a non-linear structural dynamic model through feedback. Mech Syst Signal Process 2000; 14: 637-656.

9. Gandino E and Marchesiello S. Identification of a Duffing oscillator under different types of excitation. Math Prob Eng 2010; (2010): 695025. 
10. Worden K and Tomlinson GR. Nonlinearity in experimental modal analysis. Proc Roy Soc A-Math Phy Eng Sci 2001; 359: 113-130.

11. Lee YS, Vakakis AF, McFarland DM, et al. A global-local approach to nonlinear system identification: A review. Struct Control Health Monit 2010; 17: 742-760.

12. Carrella A and Ewins DJ. Identifying and quantifying structural nonlinearities in engineering applications from measured frequency response functions. Mech Syst Signal Process 2011; 25: 1011-1027.

13. Arslan Ö, Aykan M and Özgüven HN. Parametric identification of structural nonlinearities from measured frequency response data. Mech Syst Signal Process 2011; 25: 1112-1125.

14. Noël JP, Marchesiello S and Kerschen G. Subspacebased identification of a nonlinear spacecraft in the time and frequency domains. Mech Syst Signal Process 2014; 43: 217-236.
15. Kenderi G and Fidlin A. Nonparametric identification of nonlinear dynamic systems using a synchronisationbased method. J Sound Vib 2014; 333: 6405-6423.

16. Londoño JM, Neild SA and Cooper JE. Identification of backbone curves of nonlinear systems from resonance decay responses. J Sound Vib 2015; 348: 224-238.

17. Worden K and Tomlinson GR. Nonlinearity in structural dynamics: Detection, identification and modelling. Bristol and Philadelphia: Institute of Physics Publishing, 2001. Chaps. 2-9.

18. Benhafsi Y, Penny JET and Friswell MI. A parameter identification method for discrete nonlinear systems incorporating cubic stiffness elements. Int $J$ Anal Exp Modal Anal 1992; 7: 179-195.

19. Krauss RW and Nayfeh AH. Experimental nonlinear identification of a single mode of a transversely excited beam. Nonlinear Dyn 1999; 18: 69-87.

20. Harris CM and Piersol AG. Shock and vibration handbook. 5th ed. New York: McGraw Hill, 2002. Chap. 36. 